\title{
Test yourself: right thigh mass
}

\section{Kerry L. Thomas • Evita Henderson-Jackson • Jamie T. Caracciolo}

Published online: 12 February 2015

(C) ISS 2015

\section{TEST YOURSELF:}

Question - Painful right thigh mass

Twenty-seven year-old male presents with a 6 month history of a painful right thigh mass with rapid enlargement and new onset of bleeding (Figs. 1, 2, 3 and 4)

The diagnosis can be found at doi: 10.1007/s00256-015-2113-9

K. L. Thomas $\cdot$ J. T. Caracciolo $(\bowtie)$

Department of Diagnostic Imaging, Moffitt Cancer Center,

Tampa, FL, USA

e-mail: jamie.caracciolo@moffitt.org

\section{E. Henderson-Jackson}

Department of Anatomic Pathology, Moffitt Cancer Center,

Tampa, FL, USA

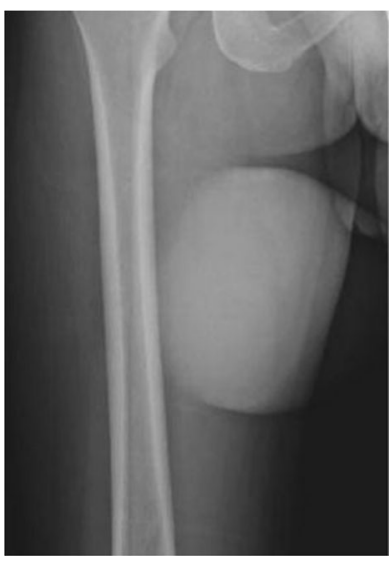

Fig. 1 AP radiograph of the right thigh
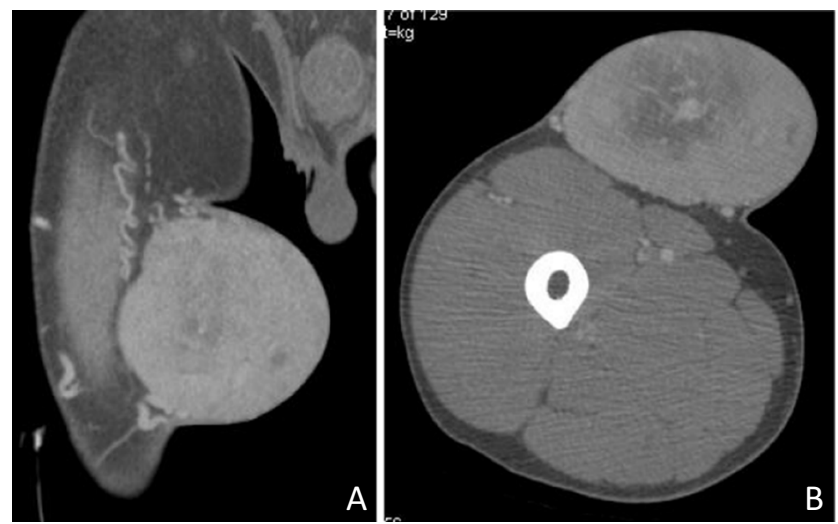

Fig. 2 (a) Coronal and (b) axial contrast-enhanced CT of the right thigh 

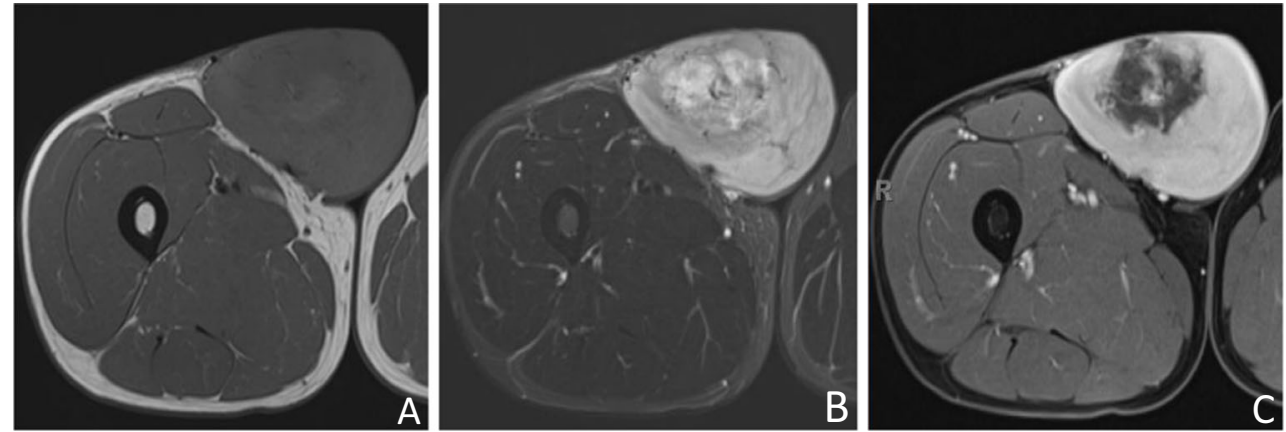

Fig. 3 Axial (a) T1-w, (b) STIR, and (c) fat-suppressed T1-w contrast-enhanced MRI of the right thigh

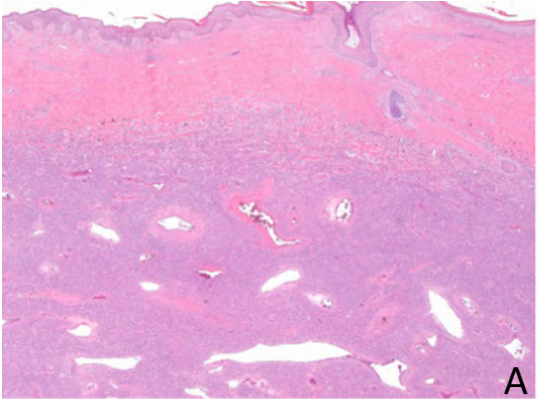

Fig. 4 Histopathological photomicrographs (hematoxylin and eosin staining) at (a) low power depicting lesion involving the subcutis and deep dermis exhibiting a monomorphic storiform pattern with

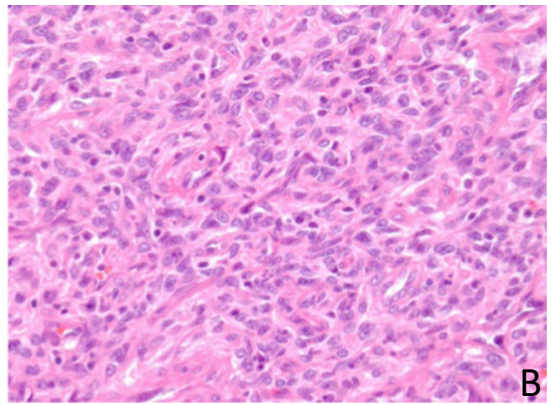

branching hemangioperictyoma-like vessels and (b) bland-appearing spindle cells with plump, ovoid to elongated vesicular nuclei and indistinct, pale eosinophilic cytoplasm (40x) 